\title{
A CHARACTERIZATION OF THE ORDERS OF REGRESSIVE $\omega$-GROUPS
}

\author{
MATTHEW J. HASSETT
}

(Received 26 February 1970, revised 21 April 1971)

Communicated by J. N. Crossley

\section{Introduction}

Let $-E, \Lambda$ and $\Lambda_{R}$ denote the collections of all non-negative integers, isols and regressive isols respectively. An $\omega$-group is a pair $(\alpha, p)$ where (1) $\alpha \subseteq E$, (2) $p(x, y)$ is a partial recursive group multiplication for $\alpha$ and (3) the function which maps each element of $\alpha$ to its inverse under $p$ has a partial recursive extension. If $G=(\alpha, p)$ is an $\omega$-group, we call the recursive equivalence type of $\alpha$ the $R E T$ or order of $G$ (written $o(G)$ ). Let $G_{R}=\left\{T \in \Lambda_{R} \mid T=o(G)\right.$ for some $\omega$-group $G\}$. It follows from the version of the Lagrange Theorem given in [4] that $\Lambda_{R}-G_{R}$ is non-empty and has cardinality $c$. In this paper we characterise the isols in $G_{R}$ as follows: A regressive isol $T$ belongs to $G_{R}$ if and only if $T \in E$ or $T$ is infinite and there exist a regressive isol $U \leqq T$ and a function $a_{n}$ from $E$ into $E-\{0\}$

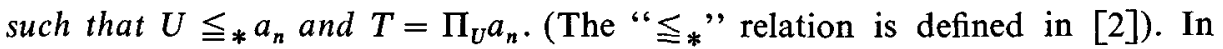
presenting the proof of this result, we shall assume that the reader is familiar with either [3] or [4]. The proof that, given $a_{n}$ and $U \leqq_{*} a_{n}$, a group of order $\Pi_{U} a_{n}$ exists is based on the natural trick - one constructs a direct product of disjoint cyclic groups of order $a_{0}, a_{1}, \cdots$ indexed by elements of a set of RET $U$. The proof that any regressive group $G$ has order of the form $\Pi_{U} a_{n}$ is trivial for finite groups; the proof for infinite regressive groups is based upon the construction of an ascending chain of finite subgroups $G_{i}$ of $G$ such that $\bigcup_{i=0}^{\infty} G_{i}=G$ and

$$
o(G)=\underbrace{o\left(G_{1}\right) \cdot \frac{o\left(G_{2}\right)}{o\left(G_{1}\right)} \cdot \frac{o\left(G_{3}\right)}{o\left(G_{2}\right)} \cdots \frac{o\left(G_{n+1}\right)}{o\left(G_{n}\right)} \cdots}_{U} .
$$

\section{Characterization of $G_{R}$}

In the following we shall write $\rho f$ to denote the range of a function $f$.

THEOREM 1. Let $G=(\tau, p)$ be an $\omega$-group of order $T$, where $T \in \Lambda_{R}$. Then 379 
there exist a regressive isol $U \leqq T$ and a function $a_{n}$ from $E$ to $E-\{0\}$ such that $U \leqq_{*} a_{n}$ and $T=\Pi_{U} a_{n}$.

Proof. We need consider only $T \in \Lambda_{R}-E$. Let $t_{n}$ be a regressive function such that $\tau=\rho t_{n}$. Let $q(x)$ be a regressing function for $t_{n}$. (We assume without loss of generality that $t_{0}$ is the identity element of $G$.) We shall give simultaneous inductive definitions of an increasing function $d(n)$, an increasing sequence $\left\{G_{n}\right\}$ of finite subgroups of $G$ such that $\bigcup_{n=0}^{\infty} G_{n}=G$ and a function $a_{n}$ such that $t_{d(n)} \leqq_{*} a_{n}$. We will then show that $v_{1} \tau-v$, where $v=\rho t_{d(n)}$, and complete the proof by showing that $T=\Pi_{v} a_{n}$, where $U=\operatorname{Re} q(v)$.

We first note that given a finite subset $\sigma$ of $\tau$ if we alternately apply $q$ and take the group closure we can effectively enumerate the smallest subgroup $G(\sigma)$ of $G$ containing $\sigma$ and closed under $q$ and the group operation. Since $\tau$ is isolated $G(\sigma)$ is finite and we can decide when $G(\sigma)$ has been obtained. We now proceed with the necessary definitions.

$$
\begin{array}{ll}
G_{-1}=\phi & \\
i=0 & \text { (1) } d(o)=1 \\
& \text { (2) } G_{0}=G\left(\left\{t_{1}\right\}\right) \\
i=n+1 & \text { (3) } a_{0}=o\left(G_{0}\right) \\
& \text { (1) } d(n+1)=(\mu y)\left[t_{y} \notin G_{n}\right] \\
& \text { (2) } G_{n+1}=G\left(\left\{t_{d(n+1)}\right\}\right) \\
& \text { (3) } a_{n+1}=\frac{o\left(G_{n+1}\right)}{o\left(G_{n}\right)} .
\end{array}
$$

It follows immediately from the definition above that both the function $d(n)$ and the sequence of sets $\left\{G_{n}\right\}$ are increasing. Given $t_{d(n)}$ one can find $t_{0}, t_{1}, \cdots, t_{d(n)}$ and determine all elements of each $G_{i}, 0 \leqq i \leqq n$. This information is sufficient for the computation of $a_{0}, a_{1}, \cdots, a_{n}$. Hence $t_{d(n)} \leqq{ }_{*} a_{n}$. Similarly given $x=t_{k} \in \tau$, there is a number $m$ such that $x \in G_{m}-G_{m-1}$ and one can use the definition above to compute $t_{d(o)}, \cdots, t_{d(m)}$. This information is sufficient to determine whether or not $x \in v$. Hence $v \mid \tau-v$.

We now prove that $o(G)=\Pi_{U} a_{n}$. We use the notation of [3]. Let $\gamma$ denote the set of all indices of finite functions $f$ such that $\delta_{e} f \subseteq v$ and $(\forall n)\left[f\left(t_{d(n)}\right)<a_{n}\right]$. Since $\operatorname{Re} q(\gamma)=\Pi_{v} a_{n}$, we need only show that $\gamma \simeq \tau$. Using Proposition 1 of [1], we shall prove this by describing a one-to-one function $\alpha(x)$ mapping $\tau$ onto $\gamma$ such that $\alpha(x)$ and $\alpha^{-1}(x)$ have partial recursive extensions. $\alpha(x)$ is defined as follows: For each $m \in E$, let

$$
\gamma_{m}=\left\{n \mid n \in \gamma \text { and } t_{d(m)} \in \delta_{e} r_{n} \text { and }\left[t_{d(k)} \in \delta_{e} r_{n} \Rightarrow k \leqq m\right]\right\} .
$$


We write $G$ and $\gamma$ as disjoint unions of finite sets as follows:

$$
\begin{aligned}
& \gamma=\gamma_{0} \cup \gamma_{1} \cup \cdots \cup \gamma_{n} \cup \cdots \\
& G=\left\{t_{0}, t_{1}, \cdots, t_{d(1)-1}\right\} \cup\left\{t_{d(1)}, \cdots, t_{d(2)-1}\right\} \cup \cdots \cup\left\{t_{d(n)}, \cdots, t_{d(n+1)-1}\right\} \cup \cdots
\end{aligned}
$$

We observe that the $n$th finite set in the decomposition of $G$ is merely $G_{n}-G_{n-1}$. It is easily seen that the cardinality of the $n$th set in each decomposition above is $\left(a_{0} a_{1} \cdots a_{n}\right)-\left(a_{0} a_{1} \cdots a_{n-1}\right)$ for $n>1$ and $a_{0}$ for $n=0$. Furthermore, given any element of either $\gamma_{n}$ or $G_{n}-G_{n-1}$, we can (uniformly) effectively recover $t_{d(n)}$ and list all elements of both $\gamma_{n}$ and $G_{n}-G_{n-1}$ in increasing order. Let $\alpha(x)$ be the function from $\tau$ to $\gamma$ which pairs the elements of each set $G_{n}-G_{n-1}$ with the elements of $\gamma_{n}$ in increasing order. The preceding discussion shows that both $\alpha(x)$ and $\alpha^{-1}(x)$ have partial recursive extensions. This completes the proof.

Let $a_{n}$ be a sequence of positive integers.

Proposition 1. If $T \leqq_{*} a_{n}$ and $T \in \Lambda_{R}-E$, then $\Pi_{T} a_{n} \in \Lambda_{R}$.

Proof. Left to the reader.

THEOREM 2. Let $T \in \Lambda_{R}-E$ and let $a_{n}$ be a sequence of positive integers such that $T \leqq_{*} a_{n}$. Then there exists a regressive $\omega$-group of order $\Pi_{T} a_{n}$.

Proof. Let $B_{E}$ be the group of all permutations of $E$ which leave all but finitely many numbers fixed, and let $f \leftrightarrow f^{*}$ be any Gödel numbering of $B_{E}$ which is one-to-one and bi-effective. It was shown in [5] that $P(E)=\left\{f^{*} \mid f \in B_{E}\right\}$ is an $\omega$-group under the induced multiplication $f^{*} \cdot g^{*}=(f \circ g)^{*}$. We shall construct a subgroup of $P(E)$ of order $\Pi_{T} a_{n}$. We will use the recursive pairing function $j(x, y)$ and associated projection functions $k(x)$ and $l(x)$ defined in [1]. Let $t_{n}$ be any regressive function such that range $\left(t_{n}\right) \in T$. Let $\gamma_{n}$ denote the cyclic permutation

$$
\left(j\left(t_{n}, 0\right), j\left(t_{n}, 1\right), \cdots, j\left(t_{n}, a_{n}-1\right)\right) .
$$

Let $\left[\gamma_{n}{ }^{*}\right]$ denote the cyclic subgroup of $P(E)$ generated by $\gamma_{n}^{*}$. Let $G$ be the subgroup of $P(E)$ generated by $\left\{\gamma_{n}{ }^{*} \mid n \subseteq E\right\}$.

Since the permutations $\gamma_{n}$ move disjoint sets of numbers, $G$ is the weak direct product of the cyclic groups $\left[\gamma_{n}{ }^{*}\right]$. Thus $G$ is an $\omega$-group with non-trivial elements of the form $\prod_{i=1}^{k} \gamma_{n_{i}}^{* l_{i}}$, where $0<l_{i}<a_{n_{i}}, i=1, \cdots, k$. Let $\alpha(x)$ be the function with domain $G$ which maps $x=\prod_{i=1}^{k} \gamma_{n_{i}}^{* l_{i}}$ to the index of the finite function $f$ defined by

$$
f(x)=\left\{\begin{array}{l}
l_{i}, x=t_{n_{i}} \text { for some } i, 1 \leqq i \leqq k \\
0, x \notin\left\{t_{n_{1}}, \cdots, t_{n_{k}}\right\}
\end{array}\right.
$$

and maps the identity permutation to the constant function $f(x) \equiv 0$. It is readily seen that $\alpha$ maps $G$ one-to-one onto the set 


$$
\beta=\left\{n \mid \delta_{e} r_{n} \subseteq \rho t_{k} \text { and }(\forall k)\left[r_{n}\left(t_{k}\right)<a_{k}\right]\right\} .
$$

Since the RET of $\beta$ is $\Pi_{r} a_{n}$, we need only show that $\alpha(x)$ and $\alpha^{-1}(x)$ have partial recursive extensions to complete the proof. We leave this straightforward verification to the reader.

\title{
References
}

[1] J. C. E. Dekker, Infinite series of Isols (Proc. Sympos. Pure Math., V (1962), Amer. Math. Soc., Providence, R. I.).

[2] J. C. E. Dekker, 'The Minimum of Two Regressive Isols', Math. Zeitschr. 83 (1964), 345-366.

[3] E. Ellentuck, 'Infinite Products of Isols', Pacific J. Math. 14 (1964), 49-52.

[4] D. Ferguson, 'Infinite Products Recursive Equivalence Types', J. Symbolic Logic 33 (1968), 221-230.

[5] M. Hassett, 'Recursive Equivalence Types and Groups', J. Symbolic Logic 34 (1969), 13-20.

[6] J. Myhill, 'Recursive Equivalence Types and Combinatorial Function', Bull. Am. Math. Soc. 64 (1958), 373-376.

[7] A. Nerode, 'Extensions to Isols', Annals of Math. 73 (1961), 362-403.

\author{
Arizona State University \\ Tempe, Arizona \\ U.S.A.
}

